\title{
Hypertension from targeted ablation of chromogranin A can be rescued by the human ortholog
}

\author{
Nitish R. Mahapatra, ${ }^{1}$ Daniel T. O’Connor, ${ }^{1,2,3}$ Sucheta M. Vaingankar, ${ }^{1}$ Amiya P. Sinha Hikim, ${ }^{4}$ \\ Manjula Mahata, ${ }^{1}$ Saugata Ray, ${ }^{1}$ Eugenie Staite, ${ }^{2}$ Hongjiang Wu, ${ }^{1}$ Yusu Gu, ${ }^{5}$ Nancy Dalton, ${ }^{5}$ \\ Brian P. Kennedy, ${ }^{1}$ Michael G. Ziegler, ${ }^{1}$ John Ross Jr., ${ }^{5}$ and Sushil K. Mahata ${ }^{1,2}$

\begin{abstract}
1Department of Medicine, UCSD, San Diego, California, USA. ${ }^{2}$ VA San Diego Healthcare System, San Diego, California, USA. ${ }^{3}$ Center for Molecular Genetics, UCSD, San Diego, California, USA. ${ }^{4}$ Department of Medicine, Harbor-UCLA Medical Center,
\end{abstract} \\ Torrance, California, USA. IInstitute of Molecular Medicine, UCSD, San Diego, California, USA.
}

\begin{abstract}
The secretory prohormone chromogranin A (CHGA) is overexpressed in essential hypertension, a complex trait with genetic predisposition, while its catecholamine release-inhibitory fragment catestatin is diminished, and low catestatin predicts augmented adrenergic pressor responses. These findings from studies on humans suggest a mechanism whereby diminished catestatin might increase the risk for hypertension. We generated $\mathrm{Chga}^{-/-}$and humanized mice through transgenic insertion of a human CHGA haplotype in order to probe CHGA and catestatin in vivo. $\mathrm{Chga}^{-/}$mice displayed extreme phenotypic changes, including: (a) decreased chromaffin granule size and number; (b) elevated BP; (c) loss of diurnal BP variation; (d) increased left ventricular mass and cavity dimensions; (e) decreased adrenal catecholamine, neuropeptide $Y$ (Npy), and ATP contents; (f) increased catecholamine/ATP ratio in the chromaffin granule; and (g) increased plasma catecholamine and Npy levels. Rescue of elevated BP to normalcy was achieved by either exogenous catestatin replacement or humanization of $\mathrm{Chga}^{-/-}$mice. Loss of the physiological "brake" catestatin in $\mathrm{Chga}^{-/}$mice coupled with dysregulation of transmitter storage and release may act in concert to alter autonomic control of the circulation in vivo, eventuating in hypertension.
\end{abstract}

\section{Introduction}

Chromogranin A (CHGA), a 48-kDa acidic polypeptide $(1,2)$, is the major protein co-stored and co-released with catecholamines from secretory vesicles in adrenal medulla and postganglionic sympathetic axons (3). Catecholamine storage vesicles (or chromaffin granules) of the adrenal medulla contain remarkably high concentrations of catecholamines $(\sim 0.6 \mathrm{M}), \mathrm{ATP}(\sim 150 \mathrm{mM})$, and $\mathrm{Ca}^{2+}(\sim 40 \mathrm{mM})(4)$, as well as CHGA $(\sim 4 \mathrm{mM})$, and CHGA seems to bind both catecholamines and $\mathrm{Ca}^{2+}(5)$. CHGA is also a prohormone that gives rise to biologically active peptides such as the dysglycemic peptide pancreastatin (human $\left.\mathrm{CHGA}_{250-301}\right)(6,7)$; the antimicrobial peptides prochromacin (bovine $\mathrm{CHGA}_{79-431}$; human $\mathrm{CHGA}_{79-439}$ ) and chromacin (bovine CHGA $_{173-194}$; human CHGA $_{176-197}$ ) (8); the vasodilator (vascular smooth muscle-relaxing) vasostatin (human $\mathrm{CHGA}_{1-76}$ ) (9); and a novel fragment of CHGA (bovine $\mathrm{CHGA}_{344-364}$ or human $\mathrm{CHGA}_{352-372}$, which we have called catestatin) that acts as a potent endogenous nicotinic cholinergic antagonist to inhibit catecholamine release (10-16). Catestatin also induces release of histamine from mast cells (17) and displays potent antimicrobial activity (18).

CHGA is targeted into the regulated (stimulus-inducible) secretory pathway (19), and the trafficking domain seems to reside in the amino-terminal region of the human protein (20). It may act

Nonstandard abbreviations used: Ang I, angiotensin I; BAC, bacterial artificial chromosome; CB6, BALB/c × C57BL/6; CHGA, chromogranin A; Dbh, dopamine $\beta$-hydroxylase; DBP, diastolic BP; humanized, mouse allele replaced with the human ortholog; Npy, neuropeptide Y; PRA, plasma renin activity; PRC, plasma renin concentration; Pnmt, phenylethanolamine N-methyltransferase; Scg2, secretogranin II; SBP, systolic BP; Th, tyrosine hydroxylase; Vmat1, vesicular monoamine transporter 1. Conflict of interest: The authors have declared that no conflict of interest exists. as an "on/off switch" in neuroendocrine cells to trigger secretory granule biogenesis (21). A reduction in CHGA synthesis decreased the number of secretory granules in neuroendocrine PC12 cells; conversely, ectopic expression of CHGA in nonendocrine cells induced granule biogenesis (21).

Expression of CHGA is heritable in humans (22), and both plasma CHGA and its releasable sympathoadrenal vesicular stores are elevated in essential (genetic) (22) hypertension. Conversely, the plasma concentration of the catestatin fragment is diminished both in individuals with established hypertension and in patients' still-normotensive offspring at genetic risk of developing the disease (23). These findings on catestatin suggest that altered CHGA peptide expression has consequences very early in the pathogenesis of hypertension.

We have developed 2 mouse models to delineate the in vivo role of Chga. Our findings in the Chgar-- mouse confirm the putative functions of Chga, including its role in vesicular biogenesis, neurotransmitter storage and release, and regulation of BP. We also show that the human $C H G A$ gene is functional in mice, mimics the tissue-specific expression of Chga, and rescues the BP to normalcy. For the first time to our knowledge, it is apparent that the human gene has a definitive role in autonomic control of the circulation. The humanized transgenic mice provide a model for functional analyses of the human $C H G A$ gene.

\section{Results}

\section{Prenatal effects}

The Chga gene is ablated by homologous recombination-guided gene targeting strategy, as illustrated in Figure 1. To test the possibility of prenatal consequences of the Chga-null state, we genotyped $85 \mathrm{~F}_{1}$ newborn pups from matings of heterozygous 


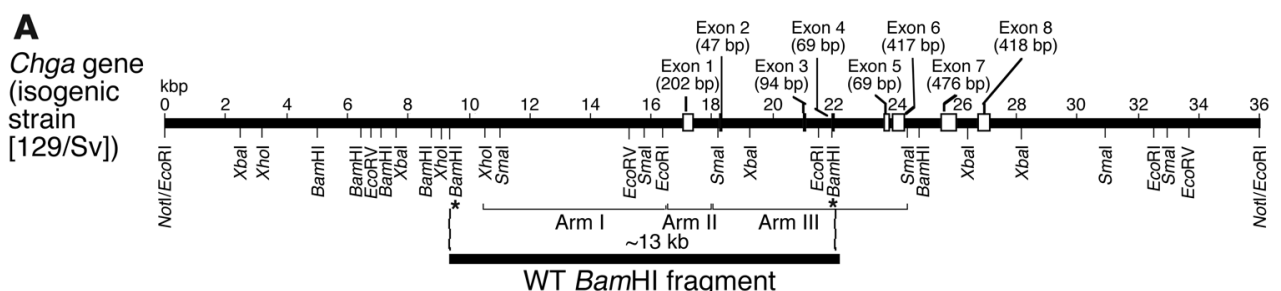

B

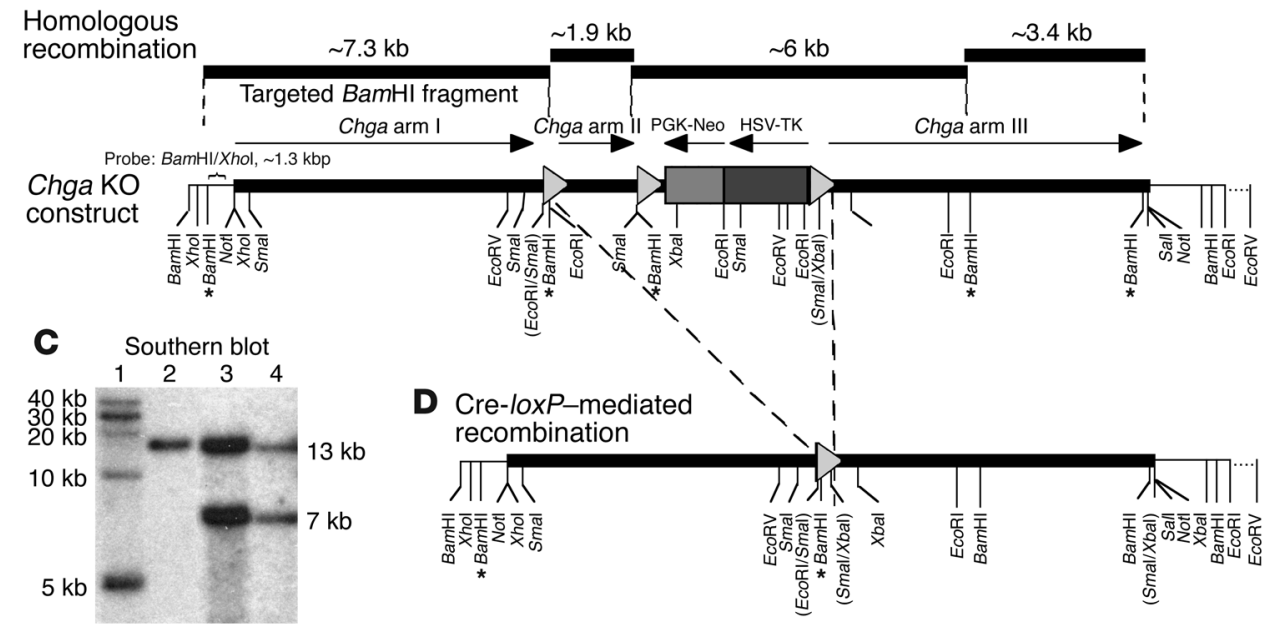

Figure 1

Chga gene targeting. (A) Chga wild-type gene structure. Chga from the isogenic mouse 129/SvJ strain showing the positions of wild-type Chga's 8 coding exons, as well as restriction sites and the locations of the 3 Chga segments (arms) used to construct the targeting vector. Also shown is an undisrupted, wild-type $\sim 13-\mathrm{kbp}$ diagnostic $\mathrm{BamHI}$ (asterisk) fragment, spanning the promoter, exons $1-3$, and corresponding introns. (B) Structure of the homologously targeted Chga gene. A Southern blot probe ( $1.3 \mathrm{~kb} \mathrm{BamHI/Xhol} \mathrm{fragment)} \mathrm{detected} \mathrm{a} \sim 7.3 \mathrm{kbp} \mathrm{BamHI}$ (asterisks) fragment after homologous recombination. (C) Southern blot results. While the probe $(\mathbf{B} ; \sim 1.3 \mathrm{kbp} \mathrm{BamHI} / \mathrm{Xhol}$ fragment) detected only a $\sim 13 \mathrm{kbp}$ fragment in wild-type DNA (A), it detected both $\sim 13-\mathrm{kbp}$ (wild-type) and 7.3-kbp (targeted) BamHI fragments in ES cell genomic DNA after homologous recombination disrupted 1 Chga allele. Lane 1: 1-kbp extension ladder; lane 2: wild-type ES cells (13-kbp band only); lane 3: clone B7 ES cells with homologous recombination in 1 Chga allele; lane 4: clone C5 cells with homologous recombination in 1 Chga allele. (D) Chga gene after homologous integration of the targeting construct into mouse genomic DNA (Chga locus on mouse chromosome 12), followed by Cre-loxP-mediated recombination (type I deletion, between the first and third loxP sites; dashed line). The triangles represent loxP recognition sites. Asterisks indicate $\mathrm{Bam} \mathrm{HI}$ sites flanking the diagnostic 7.3-kbp BamHI fragment.

$\mathrm{F}_{0}$ mice $\left(\mathrm{Cbga}^{+/-} \times \mathrm{Cbga}^{+/-}\right)$, with the following result: $30 \mathrm{Cbga}^{+/+}$, $47 \mathrm{Chga}^{+/}$, and $8 \mathrm{Chga}^{-/}$offspring. Depletion in $\mathrm{Chga}^{-/-}$homozygotes was evaluated by $\chi^{2}$ analysis (observed versus expected), which yielded $\chi^{2}=7.72(P=0.021)$, suggesting partial prenatal lethality in the $\mathrm{Cbgal}^{-/}$genotype.

\section{Verification of Chga expression ablation in vivo}

Ablation of adrenal Chga expression at the protein level was confirmed by immunoblot analysis (Figure 2A). Quantitative RT-PCR failed to detect the Chga message in endocrine or neuroendocrine tissues of $\mathrm{Chga}^{-1}$ mice (Figure 3A; adrenal gland) and revealed an approximately 64\% decrease in mRNA level in $\mathrm{Chga}^{+} /$- heterozygotes compared with $\mathrm{Chga}^{+/+}$mice (Figure 3A).

\section{Expression of other sympathochromaffin genes}

At the protein level, expression of the soluble catecholamine storage vesicle proteins Chgb and dopamine $\beta$-hydroxylase
(Dbh) was decreased in adrenal gland: Chgb by approximately $79 \%$ (Figure 2B) and Dbh by approximately $65 \%$ (Figure $2 \mathrm{C}$ ). By contrast, quantitative RTPCR analysis in the adrenal gland of $\mathrm{Chga}^{-/-}$mice (Figure 3) detected increased mRNA transcripts of other soluble proteins of chromaffin granules: Chgb (by 188\%; Figure 3B) and secretogranin II (Scg2) (by $\sim 190 \%$; Figure 3C). Increments in mRNA levels for 2 chromaffin granule membrane proteins were also seen in $\mathrm{Chga}^{-/-}$mice: vesicular (membrane) monoamine transporters vesicular monoamine transporter 1 (Vmat1) (by $\sim 165 \%$; Figure $3 \mathrm{D}$ ) and Vmat2 (by $\sim 178 \%$; Figure 3E). Results for catecholamine biosynthetic enzymes were heterogeneous: the mRNA for tyrosine bydroxylase (Th), the rate-limiting enzyme in catecholamine biosynthesis, was elevated (by $\sim 150 \%$; Figure 3F) in $\mathrm{Chga}^{-/-}$ mice, while the greatest increment in mRNA was seen for $D b b$ (by $202 \%$; Figure 3G) in Chga $a^{-/-}$ mice. In contrast, no change in phenylethanolamine N-methyltransferase (Pnmt) mRNA level was detected between wild-type $\left(\mathrm{Cbga}^{+/+}\right)$and Chga-null $\left(\mathrm{Cbga}^{-/-}\right)$ mice (Figure $3 \mathrm{H}$ ).

\section{Morphology}

Gross anatomy. After breeding heterozygotes $\left(\mathrm{Chga}^{+-}\right)$, we found that the knockout ( $\left.\mathrm{Chga}^{-/-}\right)$ pups displayed significant growth retardation (21-day-old pup body weight: Chga ${ }^{+/+}$, $12.2 \pm 0.37$ g versus $\left.\mathrm{Chga}^{-/-}, 7.93 \pm 0.25 \mathrm{~g} ; P<0.0001\right)$ for the initial 4-5 weeks after birth, followed by rapid growth, later becoming indistinguishable from wild-type littermates by 7-9 weeks of age (42-day-old pup body weight: $\mathrm{Chga}^{+/+}, 22.2 \pm 0.88$ g versus Chga $\left.^{-/}, 19.93 \pm 0.85 \mathrm{~g} ; P=0.09\right)$.

Microscopic chromaffin cell anatomy. Striking decreases in the number, size, and electron density of chromaffin granules were noted in $\mathrm{Chga}^{-/-}$animals (Figure 4 and Table 1). The volume of an average chromaffin cell in the wild-type mice was 2,508.7 $\pm 290.8 \mu \mathrm{m}^{3}$. The secretory granules in wild-type chromaffin cells occupied a volume of approximately $150 \mu \mathrm{m}^{3} /$ cell, which constituted about $6.7 \%$ of the total cytoplasmic volume. Although there was no difference in the overall cell volume in $\mathrm{Chga}^{-/-}$mice, the total chromaffin granule volume $\left(\mu \mathrm{m}^{3} / \mathrm{cell}\right)$ was reduced by approximately $47.3 \%(P<0.05)$. We also noted a significant decrease in the average granule diameter (by $\sim 24.3 \%$ : $136.2 \pm 12.6$ versus $103.1 \pm 3.66 \mathrm{~nm}$; 

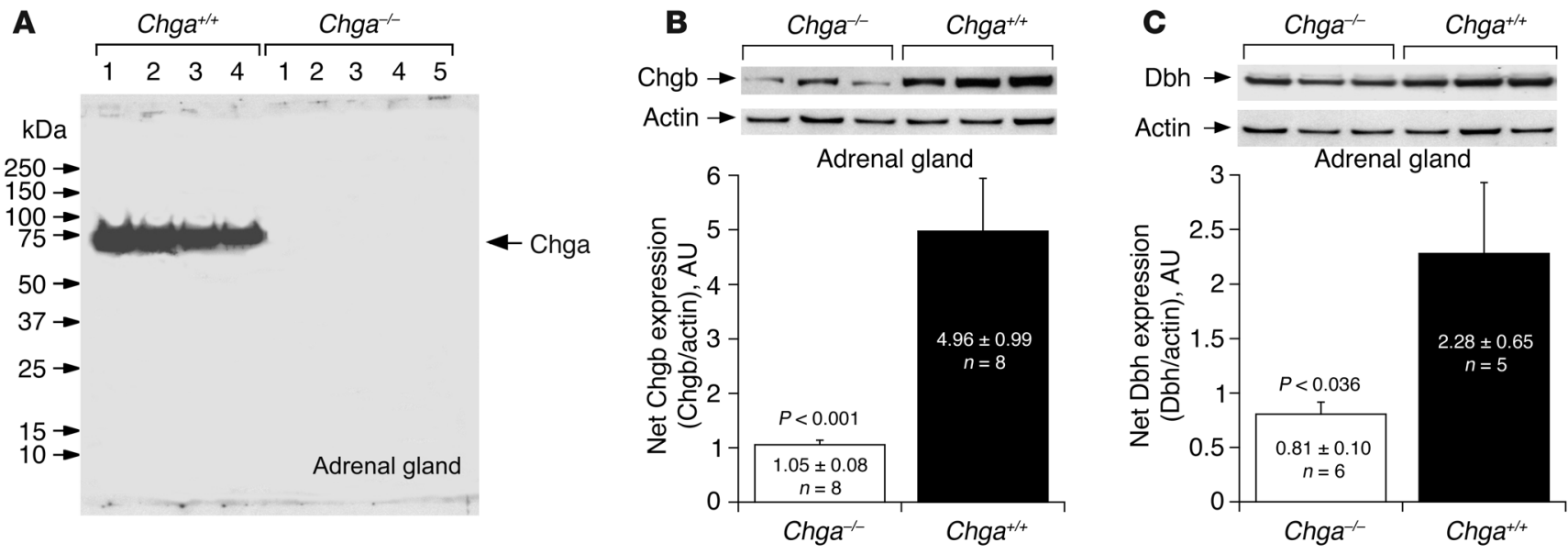

Figure 2

Verification of Chga, Chgb, and Dbh protein expression in vivo. Immunoblot analysis of Chga, Chgb, and Dbh proteins in adrenal gland homogenates from wild-type (Chga $\left.{ }^{+/+} ; n=4\right)$ and knockout $\left(\mathrm{Chga}^{-/-} ; n=4\right)$ mice. Ten micrograms of adrenal protein was loaded in each lane. (A) Chga (goat polyclonal antiserum). (B) Chgb (goat polyclonal antiserum). (C) Dbh (rabbit polyclonal antiserum). As a control, expression of $\beta$-actin (goat polyclonal) was measured in each sample.

$P<0.05)$, as well as in the granule numerical density per unit cross-sectional area (by $\sim 41.3 \%: 57.1 \pm 8.6$ versus $33.5 \pm 2.8$ granules $\left./ 10 \mu \mathrm{m}^{2} ; P<0.05\right)$ in $\mathrm{Chga}^{-/-}$mice. Chromaffin granules of the epinephrine versus norepinephrine varieties may differ in electron density (24); we did not separately study such granule types in the $\mathrm{Chga}^{+/+}$and $\mathrm{Chga}^{-/-}$mice but instead randomly sampled chromaffin cells from at least 100 micrographs (>15/mouse) to arrive at these conclusions.
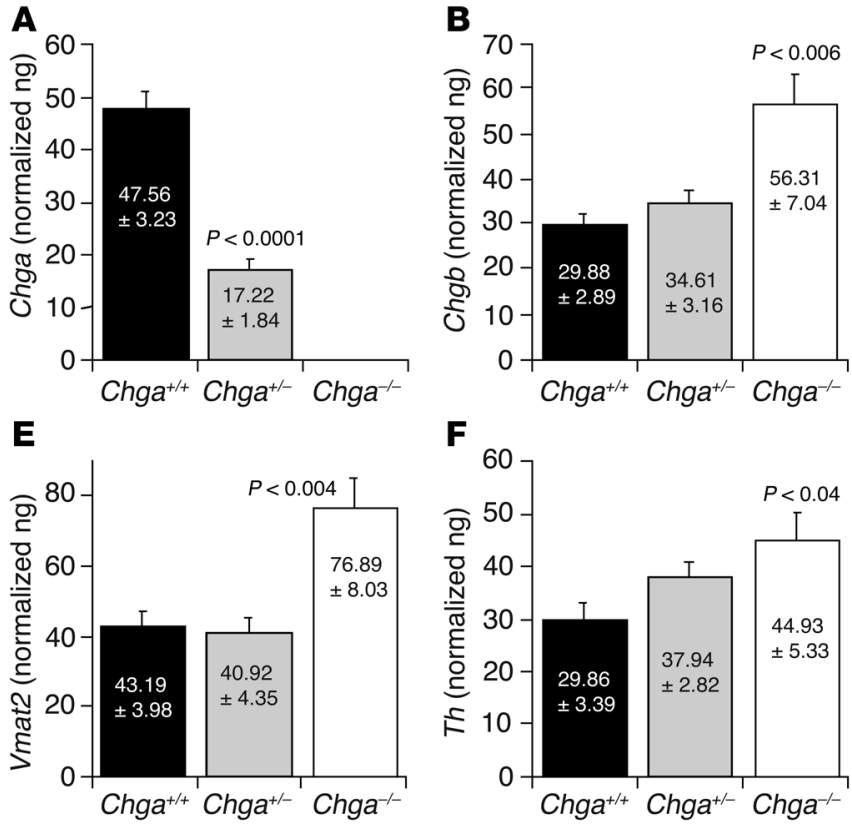

\section{Biochemical consequences}

Catecholamines: decreased in adrenal gland but increased in plasma of Chga-/mice. Using a sensitive radioenzymatic assay, we found that catecholamine levels were significantly decreased in the adrenal gland (norepinephrine by $\sim 33 \%$, epinephrine by $39 \%$ ) of $\mathrm{Chga}^{-/}$mice (Figure $5 \mathrm{~A}$ ) but significantly increased in plasma (norepinephrine by $\sim 214 \%$, epinephrine by $\sim 210 \%$; Figure 5B). Compared with $\mathrm{Chga}^{+/+}$mice, heterozygous $\left(\mathrm{Chga}^{+/-}\right)$animals also displayed decreased adrenal cat-

Figure 3

C

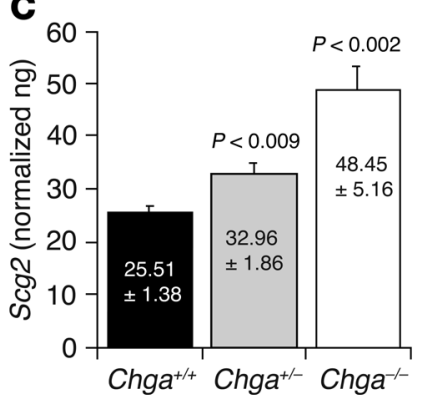

G

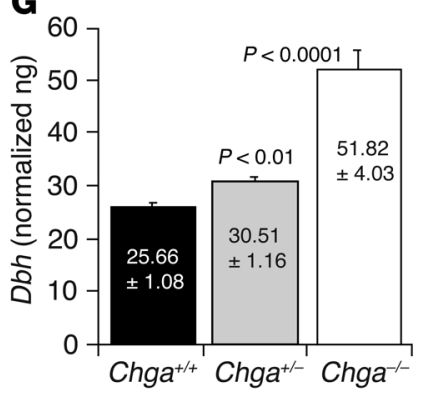

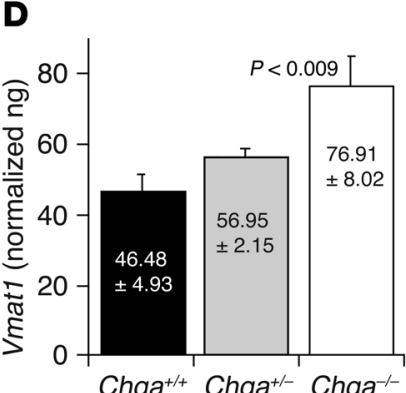

$\mathrm{Chga}^{+/+} \mathrm{Chga}^{+/-} \mathrm{Chga}^{-/-}$

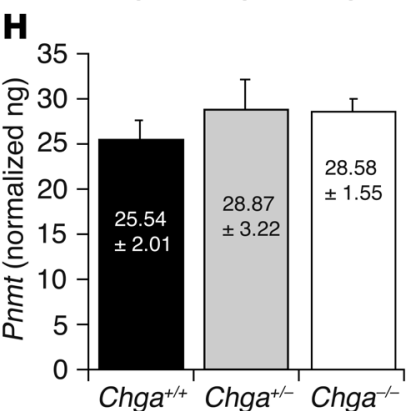

Expression of other chromaffin cell genes. Quantitative RT-PCR analysis for mRNA expression in adrenal glands from Chga ${ }^{+/+}(n=6), C{ }^{+/-}$ $(n=6)$ and $\mathrm{Chga}^{-/-}(n=6)$ mice. Chromogranins/secretogranins (chromaffin granule soluble proteins): $(\mathbf{A})$ Chga $(n=6),(\mathbf{B}) \mathrm{Chgb}(n=6)$, and (C) Scg2 $(n=6)$. Chromaffin granule membrane proteins: (D) Vmat1 $(n=6)$ and (E) Vmat2 $(n=6)$. Catecholamine biosynthetic enzymes: (F) Th $(n=6),(\mathbf{G}) \operatorname{Dbh}(n=6)$, and $(\mathbf{H})$ Pnmt $(n=6)$. Five nanograms cDNA was used for each probe. Five nanograms adrenal cDNA was used for each amplification. Results were normalized to $18 \mathrm{~S}$ ribosomal RNA (rRNA) amplified from the same sample. 


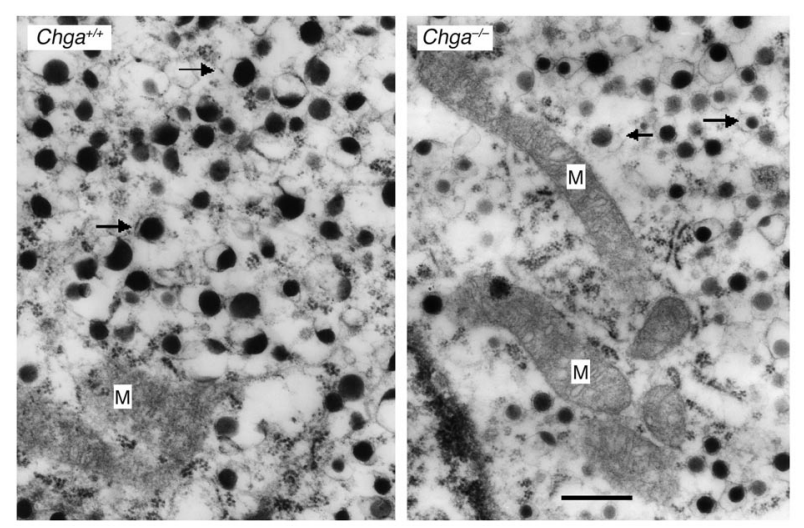

Figure 4

Microscopic chromaffin cell anatomy. Transmission electron micrograph of the mouse adrenal medulla showing chromaffin granules (arrows) in $\mathrm{Chga}^{+/+}$and $\mathrm{Chga}^{-/-}$mice. For each group (Chga ${ }^{+/+}$and $\mathrm{Chga}^{-1-}$ ), we evaluated randomly selected chromaffin cells from at least 100 micrographs (>15/mouse). M, mitochondria. Scale bar: $0.5 \mu \mathrm{m}$. In the Chga ${ }^{-/-}$chromaffin cells, note the decreased size, number, and electron density of the granules.

echolamine levels with increased levels of plasma catecholamines, and the $\mathrm{Chga}^{+/-}$values were not significantly different from those in the Chga/- animals (Figure 5, A and B), which suggests dominance of the Chga/- catecholamine phenotypes.

Neuropeptide $Y$ : decreased in adrenal gland but increased in plasma of $\mathrm{Chga}^{-/-}$mice. Since neuropeptide Y (Npy) is co-stored and co-released with catecholamines in response to sympathoadrenal stressors, we expected that Npy levels would parallel those of catecholamines in $\mathrm{Chga}^{-/-}$mice. Consistent with this notion, we found an approximately $46 \%$ decrease in adrenal Npy level but an approximately $162 \%$ increase in plasma Npy in Chga ${ }^{-/}$mice (Figure 5, C and D).

ATP: depleted in Chga-/- adrenal gland. Because ATP is co-stored and co-released with catecholamines and Npy, we anticipated parallel changes in ATP levels in $\mathrm{Chga}^{-/-}$mice. Indeed, we noted an even more profound ( $87 \%)$ depletion of adrenal ATP level in $\mathrm{Chga}^{-/-}$mice (Figure 5E), and the catecholamine/ATP ratios were elevated by approximately 4.36- (epinephrine/ATP) to 4.91-fold (norepinephrine/ATP) (Figure 5F).

Corticosterone level: augmented in $\mathrm{Chga}^{-/-}$adrenal gland. Adrenal corticosterone level was approximately $406 \%$ higher in $\mathrm{Chga}^{-/-}$than in $\mathrm{Chga}^{+/+}$mice $(P<0.0001$; Figure 5G). Plasma potassium concentration was unchanged $(4.8 \pm 0.2$ versus $4.5 \pm 0.3 \mathrm{mEq} / \mathrm{l})$.

Renin concentration: unchanged in Chga/- plasma. Plasma renin concentration (PRC) did not differ significantly $(P=0.26)$ between wild-type (1,378 $\pm 365 \mathrm{ng}$ angiotensin I/ml/h [ng Ang I/ml/h]; $n=5)$ and $\mathrm{Chga}^{-/-}(994 \pm 143 \mathrm{ng} \mathrm{Ang} \mathrm{I/ml} / \mathrm{h} ; n=9)$ animals. Nor was there a significant difference $(P=0.66)$ in plasma renin activity (PRA) between wild-type ( $8 \pm 1.09 \mathrm{ng} \mathrm{Ang} \mathrm{I/ml/h;} n=5)$ and $\mathrm{Chga}^{-/-}(7.24 \pm 1.11 \mathrm{ng} \mathrm{Ang} \mathrm{I/ml/h;} n=9)$ mice.

\section{Autonomic/cardiovascular physiology}

BP: elevated systolic and diastolic BP in Chga-/- mice. Both systolic BP (SBP) and diastolic BP (DBP) were elevated during the day in $\mathrm{Chga}^{-/}$mice at 5-6 months of age compared with wild-type littermates, according to experiments using both the tail-cuff method in restrained mice (Figure 6A) and the telemetric/continuous (Figure 6, B and C) method in unrestrained mice. From 1300 to 1500 hours, tail-cuff SBP increased by approximately $28.5 \mathrm{mmHg}$, while telemetric SBP/DBP increased by approximately $43.7 / 26.05 \mathrm{mmHg}$ (Figure 6, B and C). $\mathrm{Chga}^{+/-}$mice displayed SBP values closer to the Chga-/- values, which indicated near-dominance of the Chgat/- SBP phenotype (Figure 6A).

Continuous BP measurements: loss of diurnal variation (non-dipping) in $\mathrm{Chga}^{-/-}$mice. Telemetric measurement of ambulatory BP in Chga/mice 5-6 months of age for 1 week revealed non-dipping of BP, with both SBP (at $133.7 \pm 1.3 \mathrm{mmHg}$ ) and DBP (at $91.7 \pm 1.2 \mathrm{mmHg}$ ) remaining elevated during the day (Figure 6, B and C). The wildtype littermates showed the usual rodent daytime (sleeping) dip in SBP (by $42 \mathrm{mmHg}$ to $100.0 \pm 1.3 \mathrm{mmHg}$ ) and DBP (by $23.4 \mathrm{mmHg}$ to $76.6 \pm 1.1 \mathrm{mmHg}$ ) (Figure 6, B and C). During sleep, SBP dipped by $42.0 \mathrm{mmHg}$ and DBP by $23.4 \mathrm{mmHg}$ (Figure 6, B and C).

Catestatin replacement: rescue from elevated $B P$ in $\mathrm{Chga}^{-/-}$mice. Treatment with the catecholamine release-inhibitory CHGA fragment catestatin (20 nmol/25 g body weight i.p.; designed to achieve an extracellular target concentration of $\sim 4 \mu \mathrm{M}$ ) resulted in a substantial reduction in the elevated SBP of $\mathrm{Chga}^{-/}$mice (Figure 6D) toward the wild-type level. Two-way, repeated-measures ANOVA revealed significant SBP effects of catestatin with regard to time $(\mathrm{F}=359.9 ; P<0.001)$, strain $\left(\mathrm{Chga}^{-/-}\right.$versus $\left.\mathrm{Chga}^{+/+} ; \mathrm{F}=14.8, P=0.009\right)$, and strain/time interaction $(\mathrm{F}=79.6 ; P<0.001)$.

Echocardiography: increased LV dimensions in Chga/- mice. High BP may result in changes in LV morphology to adapt to the changed environment (afterload), with increments in wall thickness and internal dimensions. Transthoracic echocardiography (Figure 6E) in Chga/mice approximately 5 months of age detected significant increases (compared with $\mathrm{Chga}^{+/+}$littermates) in LV wall thickness (both septal and free wall) and a pronounced increment in estimated LV mass (by $\sim 72 \% ; P<0.0029$ ). We also found increases (up to $0.76 \mathrm{~mm}$ ) in LV internal diameter (cavity size) at both end systole (by $47 \% ; P<0.001$ ) and end diastole (see Methods) (by $\sim 18 \% ; P<0.017$ ) (Figure $6 \mathrm{E}$ ). Thus, the approximately 5 -month-old Chga-/- animals seemed to exhibit LV hypertrophy in the face of substantially increased afterload, with subsequent progression to LV cavity dilation.

Human ortholog CHGA rescues elevated BP observed in $\mathrm{Chga}^{-/-}$mice. The founder no. 31 integrated the entire insert of the RP11$862 \mathrm{G} 15$ clone (the entire human CHGA gene [12,150 bp] flanked by 44,068-bp upstream and 154,651-bp downstream sequences) into its genome (Figure 7A) and displayed stable inheritance of the transgene. The expression of the human CHGA transgene in founder no. 31 mirrored that of the endogenous murine Chga gene

\section{Table 1}

Comparison of various structural parameters of chromaffin cells in $\mathrm{Chga}^{+/+}$and $\mathrm{Chga}^{-/-}$animals

\begin{tabular}{lcr} 
Parameter & Chga & \\
Cell volume $\left(V_{c}, \mu \mathrm{m}^{3} / \mathrm{cell}\right)$ & $2,508.7 \pm 290.8$ & \multicolumn{1}{c}{ Chga $^{-/-}$} \\
CG volume density & $6.67 \pm 0.36$ & $3.66 \pm .5 \pm 246.6$ \\
$\quad\left(V_{v}, \%\right.$ of the cell $)$ & & \\
CG total volume $\left(\mu \mathrm{m}^{3} /\right.$ cell $)$ & $150.0 \pm 8.0$ & $79.1 \pm 7.6^{\mathrm{A}}$ \\
CG numerical density in & $57.1 \pm 8.6$ & $33.5 \pm 2.8^{\mathrm{A}}$ \\
$\quad$ cross-sectional area & & \\
$\quad\left(\right.$ granules $\left./ 10 \mu \mathrm{m}^{2}\right)$ & & \\
CG diameter $(\mathrm{nm})$ & $136.2 \pm 12.6$ & $103.1 \pm 3.6^{\mathrm{A}}$
\end{tabular}

Values are expressed as mean \pm SEM. ASignificantly different $(P<0.05$; Student's $t$ test) from wild-type mice. CG, chromaffin secretory granule. 


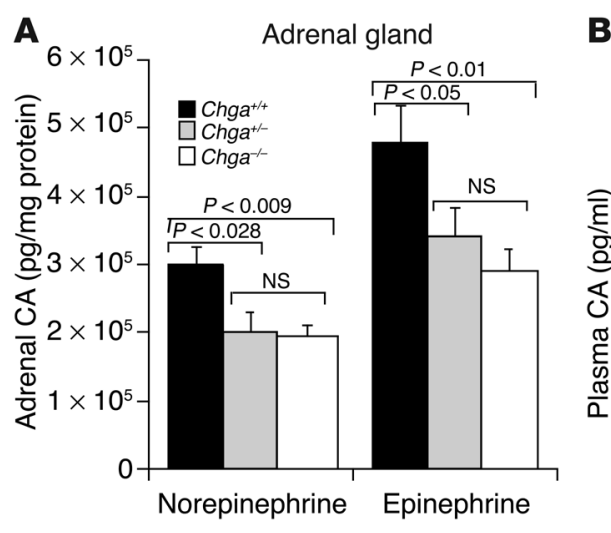

D

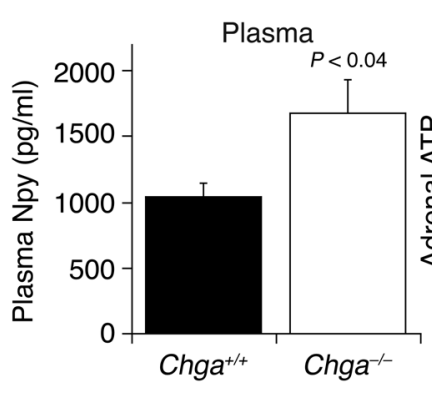

E
B

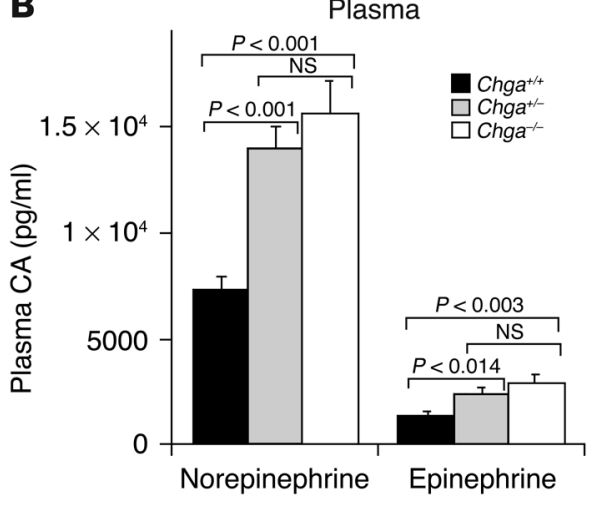

F

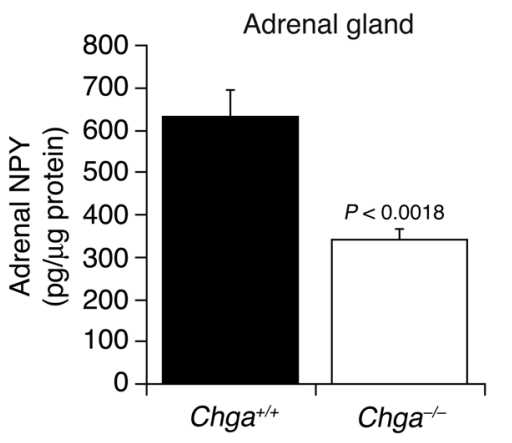

G

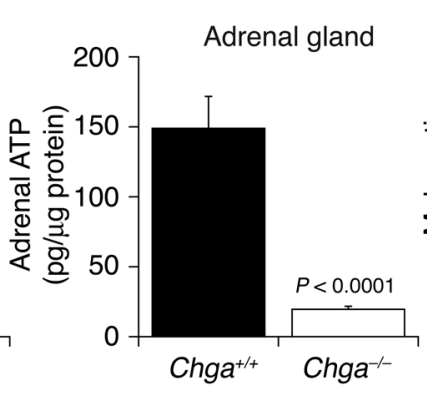

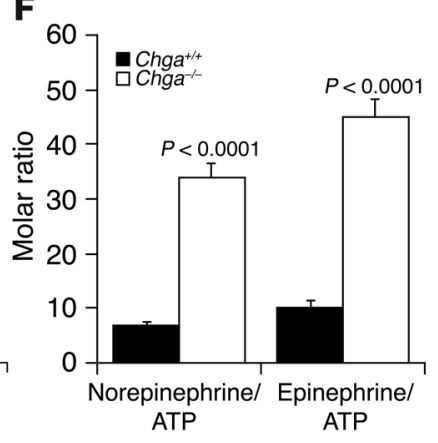

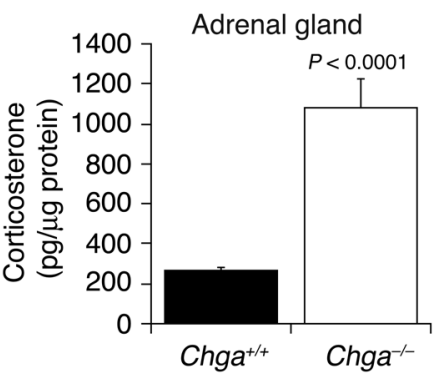

\section{Figure 5}

Biochemical consequences in $\mathrm{Chga}^{-/-}$mice. (A). Catecholamine (CA) contents in the adrenal gland of $\mathrm{Chga}^{+/+}(n=6), \mathrm{Chga}^{+/-}(n=6)$, and Chga $^{--}(n=6)$ mice as measured by a sensitive radioenzymatic assay. (B) Catecholamine concentrations in plasma of wild-type $(n=6)$, heterozygous $(n=6)$, and Chga-null $(n=6)$ mice as measured by sensitive radioenzymatic assay. (C) Adrenal NPY level in wild-type $(n=6)$ and Chga-null $(n=6)$ mice, as measured by Npy enzyme immunoassay. (D) Plasma Npy concentration in wild-type $(n=5)$ and Chga-null $(n=5)$ mice, as measured by Npy enzyme immunoassay. (E) Adrenal ATP level in wild-type $(n=10)$ and Chga-null $(n=11)$ mice, as measured by ATP luminescence assay. (F) Adrenal catecholamine/ATP molar ratios. $n=6$ adrenal glands/condition. (G) Adrenal corticosterone level in wild-type $(n=12)$ and Chga-null $(n=12)$ mice, as measured by corticosterone enzyme immunoassay.

as indicated by real-time RT-PCR (Figure 7B). As expected, nonneuroendocrine tissues showed no expression of either the $C H G A$ or the Chga gene. Both genes were expressed in the same neuroendocrine tissues. Finally, the presence of the CHGA gene rescued the elevated SBP observed in Chga-null mice (Figure 7C). This suggests that the human CHGA gene has an equivalent function to mouse Chga gene in regulating SBP. It is hoped that this mouse model $\left(\mathrm{CHGA}^{+/+} \mathrm{Chga}^{-/-}\right)$will aid in functional genomic studies in delineating the role of human $C H G A$.

\section{Discussion}

Chromaffin granule morphology. Chromogranins/secretogranins have long been proposed to control the physical process of secretory granule formation because of their $\mathrm{pH}-$, calcium-, and catecholamine-dependent aggregation properties $(1,2,5)$. However, direct evidence of the functional role(s) of CHGA in vivo has been lacking until now. In $\mathrm{Chga}^{-/-}$mice, we found approximately $41-47 \%$ decrements in both size and number of chromaffin granules (Figure 4 and Table 1). Such changes in $\mathrm{Chga}^{-/-}$mice may reflect loss of the major constituent of the chromaffin granule soluble core, Chga, with consequent impairment of formation of the granule core storage complex (5).

Recently, Kim et al. (21) proposed that Chga could act as an "on/ off switch" in neuroendocrine cells to trigger secretory granule biogenesis. In a study involving cultured cells, they found that down- regulation of Chga expression in rat PC12 pheochromocytoma cells (by antisense RNA) resulted in loss of dense-core secretory granules, impairment of stimulus-regulated secretion of a transfected prohormone, and reduction in levels of such secretory granule markers as Chgb, carboxypeptidase E, and synaptotagmin (21). Kim et al. (21) also showed that transfection of bovine CHGA (though not Chgb) into a Chga-deficient rat PC12 clone could rescue the regulated secretory phenotype and ectopic Chga expression could result in the formation of dense-core secretory granules even in cells initially without a regulated secretory pathway. Huh et al. (25) also reported decreased numbers of secretory granules in PC12 cells treated with either Chga or Chgb small interfering RNAs (25). Thus, the observed decrements in chromaffin granule number and size in $\mathrm{Chga}^{-/-}$mice (Figure 4 and Table 1) seem to confirm the role of Chga in biogenesis of dense-core secretory granules in vivo, as previously proposed in vitro by both Kim et al. (21) and Huh et al. (25).

Chromaffin cell gene expression. Quantitative RT-PCR and immunoblot studies confirmed complete ablation of Chga expression in $\mathrm{Chga}^{-/-}$mice (Figures 2A and 3A). We also noted increases in mRNA expression of other chromaffin granule soluble proteins (Chgb and Scg2; Figure 3, B and C), chromaffin granule membrane proteins (Vmat1 and Vmat2; Figure 3, D and E), and 2 catecholamine biosynthetic enzymes (Th and Dbh; Figure 3, F and G).

How might such increases in transcript expression have occurred? The transcription of chromogranins/secretogranins, as well as cat- 
A

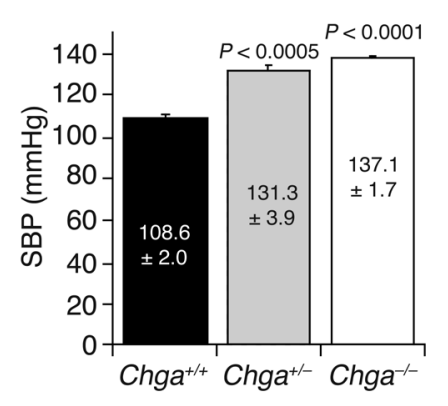

D

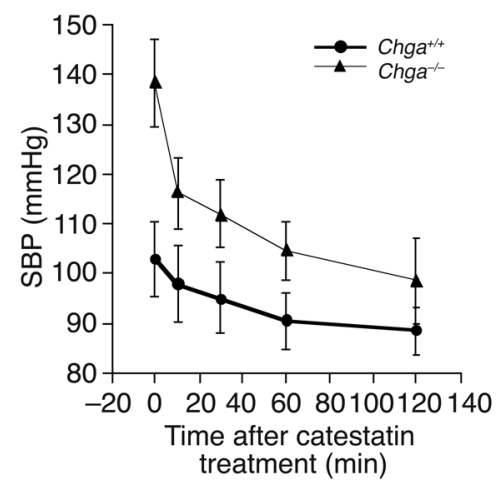

B

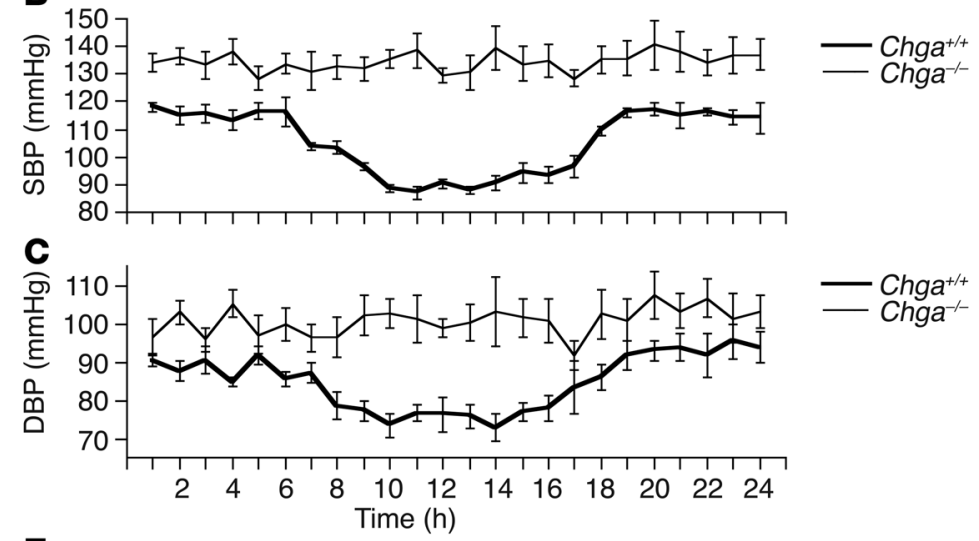

E

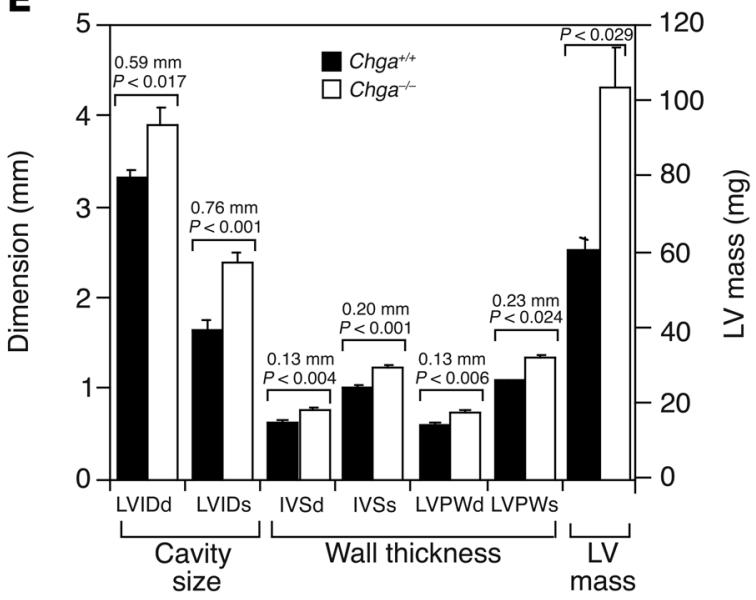

\section{Figure 6}

Autonomic/cardiovascular physiology. (A) SBP measured by the tail-cuff method in $\mathrm{Chga}^{+/+}(n=8), \mathrm{Chga}^{+/-}(n=14)$, and Chga ${ }^{-/-}(n=8)$ conscious male mice during restraint at 5-6 months of age. (B) Continuous telemetric data from unrestrained mice on SBP and DBP over a 24-hour period in $\mathrm{Chga}^{+/+}(n=5)$ and $\mathrm{Chga}^{-/-}(n=7)$ mice. (C) Telemetric data on DBP over a 24-hour period in Chga ${ }^{+/+}(n=5)$ and Chga ${ }^{-/-}$ $(n=7)$ mice. (D) Rescue from elevated SBP by the CHGA catecholamine release-inhibitory fragment catestatin: exaggerated SBP fall in $\mathrm{Chga}^{-1-}$ mice. SBP was monitored by telemetry before and after administration of catestatin $\left(20 \mathrm{nmol} / 25 \mathrm{~g}\right.$ body weight, i.p.) at time 0 in Chga ${ }^{-/-}$ $(n=4)$ and $\mathrm{Chga}^{+/+}(n=4)$ mice. Results were analyzed by 2-way, repeated-measures ANOVA, evaluating the effects of time $(\mathrm{F}=359.9$; $P<0.001)$, mouse strain $\left(\mathrm{Chga}^{+/+}\right.$vs. Chga $\left.{ }^{-/-} ; \mathrm{F}=14.8 ; P=0.009\right)$, or strain/peptide interaction $(\mathrm{F}=79.4 ; P<0.001)$. (E) Transthoracic echocardiography in $\mathrm{Chga}^{+/+}(n=8)$ and $\mathrm{Chga}^{-/-}(n=8)$ mice. LVIDd, LV dimension at end diastole; LVIDs, LV dimension at end systole; IVSd, interventricular septal thickness at end diastole; IVSs, interventricular septal thickness at end systole; LVPWd, LV posterior wall thickness at end diastole; LVPWs, LV posterior wall thickness at end systole.

echolamine biosynthetic enzymes, is augmented by preganglionic neuronal stimuli, such as acetylcholine (acting at nicotinic cholinergic receptors) (26-29) or pituitary adenylyl cyclase-activating polypeptide (28-30), in a process sometimes referred to as "stimulus-transcription coupling" or "stimulus-secretion-synthesis coupling" $(26,27,31)$, an event that is known to occur in sympathetic nerves as well as chromaffin cells (32). The $\mathrm{Chga}^{-/-}$mice displayed several signs of elevated sympathoneuronal activity, including augmented levels of plasma catecholamines (Figure 5B) and Npy (Figure 5D), increased BP (Figure 6A), and sensitivity of elevated $\mathrm{BP}$ to ganglionic blockade by catestatin (Figure 6D). Thus, transsynaptic induction (32) of these chromaffin cell transcripts (Chgb, Scg2, Th, Dbh, Vmat1, and Vmat2) is likely in the Chgal-- setting.

Another plausible mechanism for adrenal transcript induction is the approximately 4-fold elevation of adrenal glucocorticoid (rodent corticosterone) concentration in Chga ${ }^{-/-}$mice (Figure 5F), as chromogranin/secretogranin biosynthesis is augmented by glu- cocorticoids (33-35), which is especially well documented for Chga, whose glucocorticoid response element has been defined (35).

Chromaffin cell biochemistry and physiology. Since CHGA binds to catecholamines, $\mathrm{Ca}^{2+}$, and ATP (at concentrations of $\sim 0.6 \mathrm{M}, \sim 40 \mathrm{mM}$, and $\sim 150 \mathrm{mM}$, respectively) to form a storage complex $(5,36,37)$ and thereby lowers the effective osmotic pressure of the chromaffin granule interior to approximately $300 \mathrm{mOsm}$ (36), it was expected that loss of Chga in Chga/- mice would impair transmitter storage. Indeed, we found decreased chromaffin cell stores of the secretory vesicle contents norepinephrine (by 33\%) and epinephrine (by 39\%) (Figure $5 \mathrm{~A}$ ), Npy (by $\sim 46 \%$; Figure $5 \mathrm{C}$ ), Chgb (by $\sim 79 \%$; Figure $2 \mathrm{~B}$ ), Dbh (by $\sim 65 \%$; Figure $2 \mathrm{C}$ ), and especially ATP (by $\sim 87 \%$; Figure $5 \mathrm{E}$ ). Whether the transmitter storage deficit resulted directly from loss of Chga binding, indirectly from diminished granulogenesis (Figure 4 and Table 1), or both cannot be unambiguously inferred from our experiments. The catecholamine/ATP ratios were elevated by approximately 4.36-fold (epinephrine/ATP) to approximately 


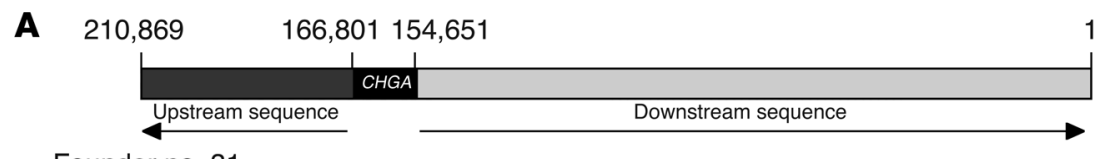

Founder no. 31
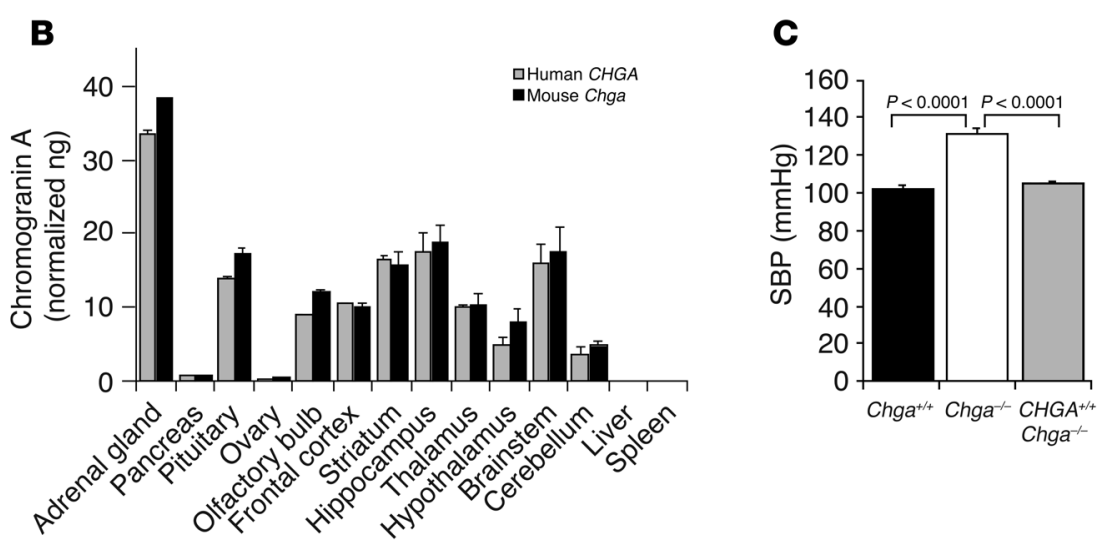

Figure 7

Characterization of the humanized CHGA mice. (A) Schematic representation of the BAC clone and founder line showing complete integration of the transgene. The BAC clone RP11862G15, a component of the human 14 contig NT_026437 spanning the locus between $87,162,128$ and $87,372,996$, has the complete CHGA (12,150 bp) gene flanked by native human sequences, 44,068 bp upstream and 154,651 bp downstream. Founder line no. 31 has the complete $210-\mathrm{kb}$ insert fragment of RP11-862G15 stably integrated in its genome. (B) RT-PCR analysis of the cDNA prepared from mouse derived from founder no. 31. The expression of both the mouse Chga and human CHGA genes was analyzed in various tissues. The tissues analyzed include adrenal gland, pancreas, pituitary, ovary, olfactory bulb, frontal cortex, striatum, hippocampus, thalamus, hypothalamus, brainstem, cerebellum, liver, and spleen. The experiment was repeated twice, and consistent results were obtained. (C) Rescue of SBP in mice humanized at the CHGA locus. SBP measured by the tail-cuff method in wild-type $\left(\mathrm{Chga}^{+/+}, n=8\right)$, null $\left(\mathrm{Chga}^{-/-}, n=8\right)$, and humanized $\left(\mathrm{CHGA}^{+/+} \mathrm{Chga}^{-/-}, n=6\right)$ conscious mice during restraint at $5-6$ months of age.

4.91-fold (norepinephrine/ATP) (Figure 5F), which indicates disruption of the chromaffin granule core storage complex (36-38) and suggests that even though both Chga and ATP are anionic (5), Chga is crucial to retention of ATP in the storage granule complex (38).

In addition to diminished transmitter storage, we also found increased chromaffin cell transmitter release, as indicated by increased plasma concentrations of norepinephrine (by $214 \%$ ) and epinephrine (by $\sim 210 \%$ ) (Figure $5 \mathrm{~B}$ ) and Npy (by $\sim 162 \%$; Figure 5D). In the absence of the catecholamine release-inhibitory catestatin fragment of Chga $(10-13,15,16)$, one would predict increased chromaffin cell transmitter release, as confirmed by our observations in plasma (Figure 5, B and D).

For both Chgb and Dbh, immunoblotting revealed decreased adrenal contents of these proteins (Figure 2, B and C), while RTPCR showed increased Chgb and Dbh transcripts (Figure 3, B and G). These findings, too, are compatible with accelerated release accompanied by a compensatory increase in transcription.

BP: bypertension, loss of diurnal variation, and catestatin rescue. Reports over the past 2 decades suggest a CHGA action in the pathogenesis of hypertension. CHGA is overexpressed in the adrenal gland in rodent genetic hypertension $(39,40)$. In human essential hypertension, both plasma CHGA concentration and its stimulus-releasable sympathoadrenal vesicular stores are elevated (22). Expression of CHGA is substantially heritable in humans (22). Conversely, the plasma concentration of the catecholamine release-inhibitory CHGA fragment catestatin is diminished both in established hypertensive individuals and in their stillnormotensive offspring at genetic risk of developing the disease, and catestatin deficiency predicts magnified pressor responses to environmental stressors (23).

Based on these findings on catestatin in humans (23), it was expected that $\mathrm{Chga}^{-/}$mice would display high BP. Consistent with this prediction, we found substantially higher $\mathrm{BP}$ in $\mathrm{Chga}^{-/}$mice (SBP by up to $43.7 \mathrm{mmHg}$ and DBP by up to $\sim 26.1 \mathrm{mmHg}$ during 1300 to 1500 hours). What caused the development of hypertension in the absence of Chga? Plasma measurements of the renin/angiotensin system revealed no systematic change in $\mathrm{Chga}^{-/}$ mice (see Results). A more likely etiologic culprit in BP elevation is loss of the physiological "braking" on transmitter release exerted by catestatin $(10,13,15)$. Elevated plasma catecholamines may act in concert with coreleased Npy (41) to elevate BP in Chga-/ mice. This interpretation is reinforced by our finding that replacement of catestatin rescues the BP elevation in Chga-/- mice (Figure 6D); since catestatin functions as a nicotinic cholinergic antagonist (autonomic ganglionic blocker) in vivo (13), the catestatin rescue of elevated BP (Figure 6D) further documents the participation of adrenergic mechanisms in the development of $\mathrm{Cbga}^{-/}$hypertension. While we did not measure plasma catecholamines after catestatin replacement in these mice, our previous work in transgenic mice documents that exogenous catestatin blocks secretagogue-induced increments of plasma catecholamines (13).

However, Chga has multiple actions, both extracellular (affecting catestatin; Figure 6D) and intracellular (affecting biogenesis of chromaffin granules; Figure 4 and Table 1), and derangement of either function could contribute to hypertension.

In cultured chromaffin cells (21), loss of Chga impairs regulated release of secretory proteins, effectively converting the regulated secretory pathway into a constitutive one. Should this phenomenon occur in vivo, one would predict increased constitutive (basal, unregulated) release of such secretory proteins as Npy. Indeed, plasma Npy was elevated (by 162\%) in Chga/- mice (Figure 5D), although the plasma Npy elevation was not out of proportion to that seen for plasma catecholamines (at 210-214\%; Figure 5B).

Finally, elevated adrenal corticosterone concentration was observed in $\mathrm{Chga}^{-/}$mice (Figure 5G). Although exogenous glucocorticoids are known to elevate BP (42) and glucocorticoid abnormalities are implicated in the pathogenesis of human hypertension (43), the mechanism underlying glucocorticoid excess in $\mathrm{Chga}^{-/-}$mice is uncertain at this time. However, several observations suggest that alterations in chromaffin cell transmitters can have profound consequences for adjacent adrenocortical cells (44-46).

Hypertension may cause LV morphology (such as wall thickness and internal dimensions) to change in adaptation to the hypertensive 
environment $(47,48)$. Transthoracic echocardiography (Figure 6E) detected significant increases in $\mathrm{Chga}^{-/-}$mice (compared with $\mathrm{Chga}^{+/+}$ littermates) at approximately 5 months of age in LV wall thickness (both septal and free wall), as well as a pronounced increment in estimated LV mass (by $72 \% ; P<0.0029$ ). We also found increases (up to $0.76 \mathrm{~mm}$ ) in LV internal diameter (cavity size), at both end systole (by $\sim 47 \% ; P<0.001$ ) and end diastole (by $\sim 18 \%, P<0.017$ ) (Figure $6 \mathrm{E}$ ). Thus, the approximately 5 -month-old $\mathrm{Chga}^{-/-}$animals seemed to exhibit LV hypertrophy in the face of substantially increased afterload, with subsequent progression to LV cavity dilation.

Continuous telemetric measurement of intraarterial BP in Chgatmice detected not only elevations of SBP (up to $\sim 43.7 \mathrm{mmHg}$ ) and DBP (up to $26.1 \mathrm{mmHg}$ ) but also loss of the usual diurnal pattern of $\mathrm{BP}$ variation, whereas the wild-type controls showed the typical rodent daytime (sleeping) dip in BP. Such loss of diurnal variation (non-dipping) in BP elevation occurs in about $25 \%$ of human hypertensives, especially in diabetics, African Americans, and patients with renal disease, where it is often defined as a nocturnal/sleeping fall of less than $10 \%$ (49). Heightened sympathetic nerve activity has been associated with non-dipping BP in humans (50). Thus, augmented release of catecholamines and Npy (especially during sleep) is likely to participate in the development of non-dipping of $\mathrm{BP}$ in the $\mathrm{Chga}^{-/-}$mice.

Rescue from elevated BP observed in Chga-null mice by bumanization of mice at the CHGA locus. The humanized mouse model for the CHGA gene offers an opportunity to examine the role of the human $C H G A$ gene in vivo in an intact animal model. Since mouse and human Chga proteins share approximately $52 \%$ identity, significant functional and regulatory differences may exist that can be examined by comparing the wild-type and humanized mouse. The expression of the human CHGA transgene mirrored that of the endogenous murine Chga gene (Figure 7B), which thereby establishes the likelihood that studying the consequences of the human transgene's expression would shed light on normal rodent physiology. Consistent with this customary expression pattern, the CHGA gene compensated for the loss of mouse Chga, at least by restoring the SBP to normalcy. We believe that this mouse model $\left(\mathrm{CHGA}^{+/+} \mathrm{Chga}^{-/-}\right)$will aid in functional genomic studies to delineate the role of $C H G A$ and its variants (14). Rescue of ablated mouse gene functions by human bacterial artificial chromosome (BAC) transgenes has also been reported in the case of $B R C A 1(51,52)$ and $B R C A 2(53)$ mice, as well as transgenic $M C 1 R$-BAC expression in $M c 1 r$-null mice (54).

Conclusions and perspectives. Although Chga has long been recognized as the heart of the transmitter storage complex in secretory granules of neurons and endocrine cells, its function in vivo has heretofore remained the topic of speculation alone. The present studies, in which Chga is ablated in intact organisms, establish a fundamental role for this protein in hormone and neurotransmitter storage and release.

\section{Methods}

\section{Gene targeting}

Mice were studied according to a protocol approved by the Animal Subjects Committee of the University of California at San Diego, and research was conducted in accordance with institutional guidelines. We obtained the CreloxP gene-targeting vector pFlox from Jamey D. Marth at UCSD (55). We isolated 2 overlapping mouse Chga genomic clones from an isogenic (mouse strain 129/Sv) genomic DNA library (in phage lambda-FIX [Stratagene]; from the Burnham Institute, La Jolla, California, USA) to maximize the likelihood of successful homologous recombination (Figure 1). Three arms from the isogenic Chga genomic DNA were inserted into the pFlox vector (Figure 1).

ES cells (strain CJ7, derived from 129/Sv mice) were transfected with 40 $\mu \mathrm{g}$ of the linearized targeting construct by electroporation $(450 \mathrm{~V}, 250 \mu \mathrm{F})$. After initial, single (positive) selection by the aminoglycoside G-418 (geneticin, $0.3 \mathrm{mg} / \mathrm{ml}$ ), 192 positive (that is, G-418-resistant) foci were selected and cloned. Screening for positive clone was done by Southern blot analysis. While a 1.3-kbp Bam HI/XhoI probe detected an approximately 13-kbp $B a m H I$ fragment from the genomic DNA of wild-type ES cells, approximately 13-kbp and approximately 7-kbp Bam HI fragments were detected in the genomic DNA of 2 positive clones out of the 192 foci used for screening. The positive clones were karyotyped, and 3 loxP sites were verified by PCR and cotransfected (by electroporation) with a Cre recombinase expression plasmid (55). Negative selection with gancyclovir $(2 \mu \mathrm{M})$ provided selective pressure in obtaining foci with excision of the entire selection cassette ( $P G K$-Neo plus HSV-TK) that was flanked by 2 loxP sites. Resulting foci were screened by 2 different sets of PCR primers, 1 set flanking each of the required 2 loxP sites in the type II (conditional) deletion (55). The type I (final) deletion excises all of exon 1 and approximately $1.5 \mathrm{kbp}$ of proximal promoter, thereby completely inactivating the Chga allele.

Successfully targeted and deleted ES cell clones were identified by PCR. The desired ES cells were microinjected ( $\sim 10$ cells/blastocyst) into host blastocysts obtained from the mouse strain C57BL/6J (black, non-agouti). After implantation (into pseudopregnant Swiss albino CD1 females), gestation, and weaning, chimeric mice were identified by coat pigmentation chimerism.

The test for germ-line transmission of the targeted transgene was production of offspring of the appropriate coat color on backcross to the wild-type strain (C57BL/6J). Germ-line male and female heterozygotes (Chga ${ }^{+/-}$; background strain $129 / \mathrm{SvJ}$ ) were mated to yield an $\mathrm{F}_{1}$ generation, approximately one-fourth of which was homozygous for type I or type II deletions. As indicated by PCR on tail DNA, $F_{1}$ pups' diploid Chga genotypes were characterized as: wild-type $\left(\mathrm{Chga}^{+/+}\right)$, heterozygous $\left(\mathrm{Chga}^{+/-}\right)$, or homozygous null $\left(\mathrm{Chga}^{-/-}\right)$.

Animals used for these studies were littermates generated by $\mathrm{F}_{1}$ cross of heterozygote $\left(\right.$ Chga $\left.^{+/-}\right)$mice, which yielded litters with $\mathrm{Chga}^{+/+}$, Chga ${ }^{+/}$, and $\mathrm{Chga}^{-/-}$mice. Chga $\mathrm{a}^{-/}$mice were later inbred for 7 generations to establish a homogeneous $\mathrm{Chgar}^{-/-}$mouse line.

\section{Generation of humanized CHGA mice}

The 210,869-bp insert of the BAC clone RP11-862G15, a component of the human 14 contig NT_026437 spanning the locus between 87,162,128 and $87,372,996$ bp, was obtained from BACPAC Resources (Children's Hospital Oakland Research Institute). This clone has the complete CHGA gene $(12,150 \mathrm{bp})$ flanked by native human sequences, $44,068 \mathrm{bp}$ upstream and 154,651 bp downstream (Figure 7A). The CHGA coding region and promoter were sequenced and confirmed to represent the wild-type allele. Amplification of the CHGA gene and the flanking sequences and sequencing of the PCR products were carried out using oligonucleotide primers. No rearrangements were detected during cloning and amplification. The primers were specific for the human gene, since they failed to amplify mouse genomic DNA, and were therefore effective for screening of transgenic mice.

The supercoiled BAC DNA, free of E. coli genomic DNA, was prepared first using a QIAGEN Large-Construct Kit according to the manufacturer's instructions. The DNA pellet obtained at the end of the preparation was resuspended in $10 \mathrm{mM}$ tris-HCl, $1 \mathrm{mM}$ EDTA, $\mathrm{pH} 8.0$ and subjected to overnight cesium chloride density gradient centrifugation using a vTi80 rotor at $305,214 \mathrm{~g}$ at $18^{\circ} \mathrm{C}$. The bottom band representing super-coiled DNA was isolated and subjected to extraction with $\mathrm{NaCl}$-saturated butanol to remove ethidium bromide, ethanol precipitated, and washed with $70 \%$ ethanol. The dried pellet was gently resuspended in injection buffer (10 mM Tris-HCl, pH 7.5, 0.1 mM EDTA, pH 8.0, and $100 \mathrm{mM} \mathrm{NaCl}$ ) and 
used for generation of BAC transgenic mice by microinjection of the construct into single-cell, fertilized $\mathrm{F}_{1}$ zygotes of BALB/c $\times$ C57BL/6J (CB6) hybrid mice. Of the 58 pups generated, only 6 had integrated BAC DNA as indicated by tissue tail genomic DNA PCR analysis. Of these six, 3 had the entire $\mathrm{BAC} C H G A$ gene integrated into their genomes. The founder mouse no. 31 had the entire $C H G A$ gene and the entire upstream and downstream sequences present in the BAC insert and displayed germ-line transmission. Southern blot analysis confirmed integration of 1 copy of the transgene.

Littermates of the $\mathrm{CHGA}^{+/-}$line derived from founder no. 31 (CB6 background) were mated for 4 generations to achieve the homozygous $\mathrm{CHGA}^{+/+} \mathrm{Chga}^{+/+}$state on a $\mathrm{CB} 6$ background. $\mathrm{CHGA}^{+/+} \mathrm{Chga}^{+/+}$mice (CB6 background) were mated with $\mathrm{Chga}^{-/}$mice $(\mathrm{C} 57 \mathrm{BL} / 6 \mathrm{~J} \times 129 / \mathrm{SvJ}$ background) to generate $\mathrm{CHGA}^{+/-} \mathrm{Chga} a^{+-}$mice. Littermate breeding produced the $\mathrm{CHGA}^{+/+} \mathrm{Chga}^{-/-}$mice humanized for the CHGA locus on a mixed $\mathrm{C} 57 \mathrm{BL} / 6 \mathrm{~J} \times 129 / \mathrm{SvJ} \times \mathrm{BALB} / \mathrm{c}$ background.

\section{Verification of gene targeting in vivo}

mRNA expression: real-time RT-PCR. Real-time RT-PCR assays of the following transcripts expressed in adrenal glands of $\mathrm{Chga}^{+/+}$and $\mathrm{Chga}^{-/-}$mice were carried out using coding region-specific primers as described previously (56): Chga (as a negative control), Chgb, Scg2, Dbh, Pnmt, Th, Vmat1, and Vmat2. Five nanograms of adrenal cDNA was amplified in each case. Results were expressed as the ratio to values determined for $18 \mathrm{~S}$ ribosomal RNA (rRNA), which was used as an RNA quantity/quality control in parallel amplifications.

The expression profile of both CHGA and Chga genes was analyzed in various tissues (founder no. 31) by real-time RT-PCR using coding regionspecific primers as described above for the Chga gene. The following primer pairs were used to specifically detect only the human CHGA: forward primer, 5'-CTGAAAGAGGCGGTGGAAGA and reverse primer, 5'-GAGAAAAGTGGTGAAGCCACAGA. Results were expressed as the hratio to values determined from $18 \mathrm{~S}$ rRNA.

Protein expression: immunoblot assay. We studied the expression of Chga, Chgb, and Dbh at the protein level in $10 \mu \mathrm{g}$ protein from adrenal gland homogenates of $\mathrm{Chga}^{+/+}$and $\mathrm{Chga}^{-/-}$mice using the following antibodies from Santa Cruz Biotechnology Inc.: Chga (goat polyclonal epitope mapping the C-terminal 20 amino acid region of human Chga), Chgb (goat polyclonal corresponding to the C-terminal 19 amino acid region of human Chgb), Dbh (rabbit polyclonal epitope mapping the C-terminal 391-630 amino acid region of human Dbh), and chemiluminescent detection. As a control, expression of $\beta$-actin (goat polyclonal corresponding to C-terminal 11 amino acid of human actin) was measured in each sample.

\section{Morphology}

Tissue preparation. Adrenal glands from $6 \mathrm{Chga}^{+/+}$and $8 \mathrm{Chga}^{-/-}$animals were removed and fixed with $5 \%$ glutaraldehyde in $0.05 \mathrm{M}$ sodium cacodylate buffer, postfixed in 1\% osmium tetroxide, and embedded in epon 812 as described previously (57). Thin sections from selected tissue blocks were cut with an LKB ultramicrotome, stained with uranyl acetate and lead citrate, and examined with a Hitachi H-600 electron microscope (57).

Morphometry. A conventional stereological technique was used to determine chromaffin cell volume in glutaraldehyde-fixed, epoxy-embedded, toluidine blue-stained adrenal sections (57). Cell volume was determined at $\times 1000$ magnification using an American Optical microscope. The volume of an individual nucleus $\left(V_{\mathrm{n}}\right)$ was calculated from the mean diameter (D) of the nucleus by direct measurements of its largest cross-sectional profiles in serial sections using the equation $V_{n}=1 / 6 \pi \mathrm{D}^{3}$. The volume of an average chromaffin cell $\left(V_{c}\right)$ was calculated from its nuclear volume $\left(V_{\mathrm{n}}\right)$ and the volume density of the nucleus $\left(V_{\mathrm{vn}}\right)$ within the cell using the equation $V_{\mathrm{c}}=V_{\mathrm{n}} / V_{\mathrm{vn}}$. The $V_{\mathrm{vn}}$ was obtained using the point-counting method $(58,59)$, by dividing the number of points falling on the nuclei by the total number of points lying over the chromaffin cells (nucleus and cytoplasm). Point counting was carried out using a square lattice grid containing 121 test points fitted to an eyepiece. The cytoplasmic volume $\left(V_{c y}\right)$ was then calculated by subtracting $V_{\mathrm{n}}$ from $V_{\mathrm{c}}$.

The point-counting method $(58,59)$ was also used to estimate the volume density and the absolute volume of the chromaffin granules by superimposing a transparent overlay bearing a double-lattice grid on electron micrographs of chromaffin cells. The chromaffin granules' $V_{\mathrm{v}}$ (which is the volume of a given cellular component per unit volume of the cytoplasm) was obtained by dividing the sum of points falling on the secretory granules by the total number of points counted over the chromaffin cell cytoplasm. Values were expressed as percentages of the chromaffin cell cytoplasmic volume occupied by chromaffin granules $\left(V_{v}, \%\right)$, obtained by multiplying the volume densities by 100 . The total volume of the secretory granules was then calculated by multiplying its volume density by the chromaffin cell cytoplasmic volume.

Numerical density of the secretory granules was determined according to an unbiased 2-dimensional rule (59) by counting the number of granules within a reference area encompassing $10 \mu \mathrm{m}^{2}$ of the chromaffin cell cytoplasm, after superimposing a transparent overlay bearing a lattice grid onto electron micrographs of the cells. Only granule profiles intersected by inclusion edges (upper and right borders) and within the frame were counted.

For each group ( $\mathrm{Chga}^{+/+}$and $\left.\mathrm{Chgaa^{-/ }}\right)$, we evaluated randomly selected chromaffin cells from at least 100 micrographs (>15/mouse).

\section{Biochemical consequences}

Catecholamines in plasma and adrenal gland. Blood was collected through a transthoracic ventricular puncture while the animals were unconscious after receiving a rodent anesthesia mixture $(15 \mathrm{mg} / \mathrm{kg}$ of ketamine, $1.6 \mathrm{mg} / \mathrm{ml}$ of xylazine, and $0.3 \mathrm{mg} / \mathrm{ml}$ of acepromazine; $0.1-0.15 \mathrm{ml}$ per $25-30 \mathrm{~g}$ mouse; i.p.), and placed in ice-chilled tubes containing EDTA. Plasma was separated and stored at $-70^{\circ} \mathrm{C}$ until use. Adrenal glands were dissected and suspended in $0.5 \mathrm{ml}$ of ice-cold $10 \mathrm{mM}$ HEPES, pH 6. Clear supernatants of the adrenal homogenates were stored at $-70^{\circ} \mathrm{C}$ until assay. Plasma and adrenal catecholamines were assayed by a sensitive radioenzymatic method based on catechol-O-methyltransferase, as described previously (60).

Npy in plasma and adrenal gland. Npy in the plasma samples was quantified using $50 \mu \mathrm{l}$ in duplicate by an enzyme immunoassay kit (Bachem AG) as per the manufacturer's protocol, running a spectrum of standards along with the samples. The results were expressed as picograms of Npy/milliliter of plasma. For estimation of Npy in adrenal glands, 2 glands were harvested and homogenized in $500 \mu \mathrm{l}$ of Npy assay buffer. The purified, lyophilized homogenate samples were dissolved in $300 \mu \mathrm{l}$ Npy assay buffer and diluted 50 -fold in the same assay buffer, and $50 \mu \mathrm{l}$ of the diluted samples in duplicate were used for estimation of Npy by an enzyme immunoassay kit as per the manufacturer's protocol. Protein in each sample (before 50fold dilution) was measured using a Bio-Rad Laboratories protein assay reagent, and the results were expressed as picograms of $\mathrm{Npy} /$ micrograms of total protein in the adrenal glands.

ATP in adrenal gland. Freshly dissected adrenal glands were homogenized in $300 \mu \mathrm{l}$ of $10 \mathrm{mM}$ ice-cold $\mathrm{HCl}$ (to stabilize ATP) and centrifuged at $20,000 \mathrm{~g}$ for 25 minutes at $4^{\circ} \mathrm{C}$, and the clear supernatant was collected and stored at $-70^{\circ} \mathrm{C}$ until further use. Twenty-five microliters of the adrenal lysates were placed in clear polystyrene tubes in an ultrasensitive, low-background luminometer (AutoLumat LB 953; Berthold Technologies GmbH) with a Peltier-cooled $\left(8^{\circ} \mathrm{C}\right)$ photomultiplier tube. One hundred microliters of ATP assay buffer containing $100 \mathrm{mM}$ Tris acetate, $\mathrm{pH}$ 7.8, $10 \mathrm{mM}$ magnesium acetate, $1 \mathrm{mMEDTA}, 0.1 \mathrm{mMluciferin}, 1 \mathrm{mg} / \mathrm{mlBSA}$, and $15 \mathrm{ng} / \mathrm{ml}$ QuantiLum recombinant luciferase (Promega) were injected into each 
tube, and flash luminescence was recorded as RLU for 10 seconds. Ten millimolar $\mathrm{HCl}$ alone (without adrenal homogenate) was used as an assay blank. With a standard curve, RLU values were converted to ATP concentrations $(\mathrm{ng} / \mu \mathrm{l})$, normalized to protein concentrations, and expressed as nanograms/micrograms of total protein.

Corticosterone concentrations in adrenal gland. We determined corticosterone (the rodent glucocorticoid) concentrations in adrenal homogenates using a Correlate-EIA Corticosterone Enzyme Immunoassay Kit (Assay Designs) in accordance with the manufacturer's protocol. Corticosterone concentrations were normalized to protein concentrations in the samples and expressed as picograms/micrograms of total protein.

PRC and PRA. Blood was collected on ice into EDTA ( $1 \mathrm{mg} / \mathrm{ml}$ blood). The plasma was separated and frozen at $20^{\circ} \mathrm{C}$ until assayed. Plasma Ang I was measured using the GammaCoat Plasma Renin Activity ${ }^{125}$ I-RIA Kit (catalog CA1533; DiaSorin). PRC (in the presence of exogenous rennin substrate) was determined by diluting plasma (1:5) with $\times 1$ maleate buffer. One microliter of diluted plasma was incubated with $14 \mu$ lof dialyzed rat renin substrate plus 7 $\mu \mathrm{l}$ maleate buffer. This mixture was further diluted with $2.2 \mu \mathrm{l}$ of maleate generation buffer and $0.22 \mu \mathrm{l}$ of PMSF solution according to the manufacturer's recommendations. After removal and freezing of $10 \mu \mathrm{l}$ for background determination at $4^{\circ} \mathrm{C}$, the remaining volume was incubated for 1 hour at $37^{\circ} \mathrm{C}$. PRA (in the absence of exogenous renin substrate) was determined using $12 \mu \mathrm{l}$ of plasma plus $1.2 \mu \mathrm{l}$ of maleate generation buffer and $0.12 \mu \mathrm{l}$ of the PMSF solution. Five microliters was removed and frozen as the $4^{\circ} \mathrm{C}$ background sample. The rest was incubated for one hour at $37^{\circ} \mathrm{C}$. At the end of the incubation time, the sample was treated as described above. The assay was performed on $5 \mu \mathrm{l}$ of the incubated sample. Again, the $4^{\circ} \mathrm{C}$ sample values were subtracted, and values were adjusted for dilutions.

\section{Autonomic/cardiovascular physiology}

Noninvasive tail-cuffmeasurement of BP. BP of wild-type $\left(\mathrm{Chga}^{+/+}\right)$, heterozygous $\left(\mathrm{Chga}^{+/-}\right)$, null $\left(\mathrm{Chga}^{-/-}\right)$, and humanized $\left(\mathrm{CHGA}^{+/+} \mathrm{Chga}^{-/-}\right)$mice were measured using the BP-2000 Blood Pressure Analysis System (Visitech Systems Inc.), which employs noninvasive tail-cuffs. Mice were placed into individual rodent restraint holders on a preheated specimen platform at $38^{\circ} \mathrm{C}$ as measured by a LCD strip thermometer. BP values were recorded 10 times in rapid succession and the results were averaged. This process was repeated 3-4 times daily between 1300 and 1500 hours, for 3-5 consecutive days. During measurements, data were continuously stored in a notebook computer running the BP Analysis software package (Visitech Systems Inc.) via a PCMCIA data acquisition card. Averaged readings of SBP having standard deviation of less than $10 \mathrm{mmHg}$ were accepted for further analysis.

Telemetric continuous intraarterial measurement of $B P$. For telemetric measurement of BP using the Data Sciences International (DSI; Transoma Medical) PhysioTel telemetry system, adult mice were anesthetized with isoflurane ( $5 \%$ for induction and $2 \%$ for maintenance) and implanted in the left carotid artery with a catheter coupled to a TA11PA-C20 (DSI) transmitter. Telemetry signals were received by an antenna below the cage that relayed the data to a signal processor (DataQuest A.R.T. Gold Version 2.3; DSI) connected to a Compaq desktop personal computer (Hewlett-Packard). We waited 10 days for normalization of the diurnal pattern of BP after the implantation surgery before recording $\mathrm{BP}$ in these conscious mice fitted with DSI transmitters.
Catestatin replacement. We treated $\mathrm{Chga}^{-/-}$mice with catestatin (human $\mathrm{CHGA}_{352-372} ; 20 \mathrm{nmol} / 25 \mathrm{~g}$ i.p.; designed to achieve an extracellular target concentration of $\sim 4 \mu \mathrm{M}$ ) and measured BP 10, 30, 60 and 120 minutes after the injection.

Transthoracic echocardiography. Mice were anesthetized with isoflurane (5\% for induction and $2 \%$ for maintenance). The anterior chest was shaved, and small-needle echo leads were entered through the skin on the right and left upper extremities and the left leg. The echo (Sonos 4500 Ultrasound Imaging System, model M2424A; Hewlett-Packard) employs dynamically focused symmetrical annular array technology. The $2 \mathrm{D}$ images were obtained in whiteon-black format with M-mode and spectral Doppler in black-on-white format. M-mode tracings were digitized with a scanner (ScanJet Iicx; Hewlett-Packard) with an application program (Desk Scan II; Hewlett-Packard) and visualized in $8 \times 10$-inch format to facilitate accurate measurements. The images were measured by 2 individuals using NIH Image J (version 1.31v; http://rsb.info. nih.gov/ij/) on a 15-inch computer screen by the American Society of Echocardiography Leading-Edge technique (61). End diastole and end systole interventricular septal thickness, left ventricular internal dimension, and left ventricular posterior free wall thickness were determined, with end diastole being defined as the maximal LV diastolic dimension and end systole as the most anterior systolic excursion of the LV posterior wall. LV mass (LVM in diastole, in $\mathrm{mg}$ ) was computed by the M-mode (cubed) method (62) as LVM $=1.05 \times$ $\left[(\text { IVS + LVID + LVPW })^{3}-(\text { LVID })^{3}\right]$.

Statistical analysis. Results are expressed as mean \pm SEM. When only 2 conditions were compared, the data were evaluated by unpaired Student's $t$ tests. When multiple conditions were compared, a 1- or 2-way ANOVA test was used, followed by Dunnett's multiple comparison post-hoc tests, if appropriate. Descriptive and inferential statistics were performed with the InStat software program (GraphPad Software). $P$ values less than 0.05 were considered statistically significant.

\section{Acknowledgments}

This work was supported by grants from the Department of Veterans Affairs (to S.K. Mahata and D.T. O'Connor) and the NIH (R29 DA11311 to S.K. Mahata; DK 60702, P01 HL58120, and U01 HL69758 to S.K. Mahata and D.T. O'Connor; and HD 39293 to A.P. Sinha Hikim). We are thankful to Jurgen Schnermann for conducting the renin assay. Anthony Wynshaw-Boris provided helpful discussion on BAC haplotype strategies.

Received for publication December 30, 2004, and accepted in revised form April 19, 2005.

Address correspondence to: Sushil K. Mahata, Department of Medicine (0838), University of California San Diego School of Medicine and VA San Diego Healthcare System, 9500 Gilman Drive, La Jolla, California 92093-0838, USA. Phone: (858) 5528585, ext. 2637 or (858) 534-0639; Fax: (858) 642-6425; E-mail: smahata@ucsd.edu.

Nitish R. Mahapatra, Daniel T. O'Connor, and Sucheta M. Vaingankar contributed equally to this work.
1. Winkler, H., and Fischer-Colbrie, R. 1992. The chromogranins A and B: the first 25 years and future perspectives. Neuroscience. 49:497-528.

2. Taupenot, L., Harper, K.L., and O'Connor, D.T. 2003. Mechanisms of disease: the chromogranin-secretogranin family. N. Engl. J. Med. 348:1134-1149.

3. Takiyyuddin, M.A., et al. 1990. Is physiologic sympathoadrenal catecholamine release exocytotic in humans? Circulation. 81:185-195.
4. Winkler, H., Apps, D.K., and Fischer-Colbrie, R. 1986. The molecular function of adrenal chromaffin granules: established facts and unresolved topics. Neuroscience. 18:261-290.

5. Videen, J.S., Mezger, M.S., Chang, Y.M., and O'Connor, D.T. 1992. Calcium and catecholamine interactions with adrenal chromogranins. Comparison of driving forces in binding and aggregation. J. Biol. Chem. 267:3066-3073.
6. Tatemoto, K., et al. 1986. Pancreastatin, a novel pancreatic peptide that inhibits insulin secretion. Nature. 324:476-478.

7. Cadman, P.E., Rao, F., Mahata, S.K., and O'Connor, D.T. 2002. Studies of the dysglycemic peptide, pancreastatin, using a human forearm model. Ann. N. Y. Acad. Sci. 971:528-529.

8. Strub, J.M., et al. 1996. Antibacterial activity of glycosylated and phosphorylated chromogranin 
A-derived peptide 173-194 from bovine adrenal medullary chromaffin granules. J. Biol. Chem. 271:28533-28540.

9. Aardal, S., Helle, K.B., Elsayed, S., Reed, R.K., and Serck-Hanssen, G. 1993. Vasostatins, comprising the $\mathrm{N}$-terminal domain of chromogranin A, suppress tension in isolated human blood vessel segments. J. Neuroendocrinol. 5:405-412.

10. Mahata, S.K., et al. 1997. Novel autocrine feedback control of catecholamine release. A discrete chromogranin A fragment is a noncompetitive nicotinic cholinergic antagonist. J. Clin. Invest. 100:1623-1633.

11. Mahata, S.K., Mahata, M., Parmer, R.J., and O'Connor, D.T. 1999. Desensitization of catecholamine release: the novel catecholamine releaseinhibitory peptide catestatin (chromogranin $\mathrm{A}_{344-364}$ ) acts at the receptor to prevent nicotinic cholinergic tolerance. J. Biol. Chem. 274:2920-2928.

12. Mahata, S.K., Mahata, M., Wakade, A.R., and O'Connor, D.T. 2000. Primary structure and function of the catecholamine release inhibitory peptide catestatin (chromogranin A344-364): identification of amino acid residues crucial for activity. Mol. Endocrinol. 14:1525-1535.

13. Mahata, S.K., et al. 2003. Catecholamine secretory vesicle stimulus-transcription coupling in vivo. Demonstration by a novel transgenic promoter photoprotein reporter and inhibition of secretion and transcription by the chromogranin A fragment catestatin. J. Biol. Chem. 278:32058-32067.

14. Wen, G., et al. 2004. Both rare and common polymorphisms contribute functional variation at CHGA, a regulator of catecholamine physiology. Am. J. Hum. Genet. 74:197-207.

15. Mahata, S.K., et al. 2004. The catecholamine release-inhibitory "catestatin" fragment of chromogranin a: naturally occurring human variants with different potencies for multiple chromaffin cell nicotinic cholinergic responses. Mol. Pharmacol. 66:1180-1191

16. Mahata, S.K. 2004. Catestatin - the catecholamine release inhibitory peptide: a structural and functional overview. Current Medicinal Chemistry-Immunology, Endocrine \& Metabolic Agents. 4:221-234.

17. Kruger, P.G., Mahata, S.K., and Helle, K.B. 2003 Catestatin (CgA344-364) stimulates rat mast cell release of histamine in a manner comparable to mastoparan and other cationic charged neuropeptides. Regul. Pept. 114:29-35.

18. Briolat, J., et al. 2005. New antimicrobial activity for the catecholamine release-inhibitory peptide from chromogranin A. Cell. Mol. Life Sci. 62:377-385.

19. Kelly, R.B. 1985. Pathways of protein secretion in eukaryotes. Science. 230:25-32.

20. Taupenot, L., et al. 2002. Identification of a novel sorting determinant for the regulated pathway in the secretory protein chromogranin A. J. Cell Sci. 115:4827-4841.

21. Kim, T., Tao-Cheng, J., Eiden, L.E., and Loh, Y.P. 2001. Chromogranin A, an "on/off" switch controlling dense-core secretory granule biogenesis. Cell. 106:499-509.

22. Takiyyuddin, M.A., et al. 1995. Chromogranin A in human hypertension. Influence of heredity. Hypertension. 26:213-220.

23. O'Connor, D.T., et al. 2002. Early decline in the catecholamine release-inhibitory peptide catestatin in humans at genetic risk of hypertension. J. Hypertens. 20:1335-1345.

24. Kobayashi, S., and Coupland, R.E. 1993. Morphological aspects of chromaffin tissue: the differential fixation of adrenaline and noradrenaline. J. Anat. 183:223-235

25. Huh, Y.H., Jeon, S.H., and Yoo, S.H. 2003. Chromogranin B-induced secretory granule biogenesis: comparison with the similar role of chromogranin A.
J. Biol. Chem. 278:40581-40589.

26. Tang, K., et al. 1996. Stimulus-transcription coupling in pheochromocytoma cells. Promoter region-specific activation of chromogranin A biosynthesis. J. Biol. Chem. 271:28382-28390.

27. Tang, K., et al. 1997. Stimulus coupling to transcription versus secretion in pheochromocytoma cells. Convergent and divergent signal transduction pathways and the crucial roles for route of cytosolic calcium entry and protein kinase C. J. Clin. Invest. 100:1180-1192.

28. Mahata, S.K., et al. 1999. Neuroendocrine cell type-specific and inducible expression of the secretogranin II gene: crucial role of cyclic adenosine monophosphate and serum response elements. Endocrinology. 140:739-749.

29. Mahapatra, N.R., et al. 2000. Neuroendocrine cell type-specific and inducible expression of the chromogranin B gene: crucial role of the proximal promoter. Endocrinology. 141:3668-3678.

30. Taupenot, L., Mahata, S.K., Wu, H., and O'Connor, D.T. 1998. Peptidergic activation of transcription and secretion in chromaffin cells. cis and trans signaling determinants of pituitary adenylyl cyclaseactivating polypeptide (PACAP). J. Clin. Invest. 101:863-876.

31. Eiden, L.E., Giraud, P., Dave, J.R., Hotchkiss, A.J., and Affolter, H.U. 1984. Nicotinic receptor stimulation activates enkephalin release and biosynthesis in adrenal chromaffin cells. Nature. 312:661-663.

32. Trocme, C., Ravassard, P., Sassone-Corsi, P., Mallet, J., and Biguet, N.F. 2001. CREM and ICER are differentially implicated in trans-synaptic induction of tyrosine hydroxylase gene expression in adrenal medulla and sympathetic ganglia of rat. J. Neurosci. Res. 65:91-99.

33. Rausch, D.M., Iacangelo, A.L., and Eiden, L.E. 1988. Glucocorticoid- and nerve growth factor-induced changes in chromogranin A expression define two different neuronal phenotypes in PC12 cells. Mol. Endocrinol. 2:921-927.

34. Fischer-Colbrie, R., Wohlfarter, T., Schmid, K.W., Grino, M., and Winkler, H. 1989. Dexamethasone induces an increased biosynthesis of chromogranin A in rat pituitary gland. J. Endocrinol. 121:487-494.

35. Rozansky, D.J., Wu, H., Tang, K., Parmer, R.J., and O'Connor, D.T. 1994. Glucocorticoid activation of chromogranin A gene expression. Identification and characterization of a novel glucocorticoid response element. J. Clin. Invest. 94:2357-2368.

36. Kopell, W.N., and Westhead, E.W. 1982. Osmotic pressures of solutions of ATP and catecholamines relating to storage in chromaffin granules. J. Biol. Chem. 257:5707-5710.

37. Yoo, S.H., Albanesi, J.P., and Jameson, D.M. 1990. Fluorescence studies of nucleotide interactions with bovine adrenal chromogranin A. Biochim. Biophys. Acta. 1040:66-70.

38. Berneis, K.H., Goetz, U., Da Prada, M., and Pletscher, A. 1973. Interaction of aggregated catecholamines and nucleotides with intragranular proteins. Naunyn Schmiedebergs Arch. Pharmacol. 277:291-296.

39. Schober, M., Howe, P.R., Sperk, G., Fischer-Colbrie, R., and Winkler, H. 1989. An increased pool of secretory hormones and peptides in adrenal medulla of stroke-prone spontaneously hypertensive rats. Hypertension. 13:469-474.

40. O'Connor, D.T., et al. 1999. Catecholamine storage vesicle protein expression in genetic hypertension. Blood Press. 8:285-295.

41. Lundberg, J.M. 1996. Pharmacology of cotransmission in the autonomic nervous system: integrative aspects on amines, neuropeptides, adenosine triphosphate, amino acids and nitric oxide. Pharmacol. Rev. 48:113-178.

42. Vogt, C.J., and Schmid-Schonbein, G.W. 2001.
Microvascular endothelial cell death and rarefaction in the glucocorticoid-induced hypertensive rat. Microcirculation. 8:129-139.

43. Whitworth, J.A., Brown, M.A., Kelly, J.J., and Williamson, P.M. 1995. Mechanisms of cortisol-induced hypertension in humans. Steroids. 60:76-80.

44. Bornstein, S.R., and Ehrhart-Bornstein, M. 2000. Basic and clinical aspects of intraadrenal regulation of steroidogenesis [review]. Z. Rheumatol. 59(Suppl. 2):II12-II17.

45. Bornstein, S.R., et al. 2000. Deletion of tyrosine hydroxylase gene reveals functional interdependence of adrenocortical and chromaffin cell system in vivo. Proc. Natl. Acad. Sci. U. S. A. 97:14742-14747.

46. Ehrhart-Bornstein, M., Haidan, A., Alesci, S., and Bornstein, S.R. 2000. Neurotransmitters and neuropeptides in the differential regulation of steroidogenesis in adrenocortical-chromaffin cocultures. Endocr. Res. 26:833-842.

47. Schillaci, G., et al. 2000. Continuous relation between left ventricular mass and cardiovascular risk in essential hypertension. Hypertension. 35:580-586.

48. Okin, P.M., et al. 2004. Regression of electrocardiographic left ventricular hypertrophy during antihypertensive treatment and the prediction of major cardiovascular events. JAMA. 292:2343-2349.

49. Pickering, T.G., and Kario, K. 2001. Nocturnal nondipping: what does it augur? Curr. Opin. Nephrol. Hypertens. 10:611-616.

50. Sherwood, A., Steffen, P.R., Blumenthal, J.A., Kuhn, C., and Hinderliter, A.L. 2002. Nighttime blood pressure dipping: the role of the sympathetic nervous system. Am. J. Hypertens. 15:111-118.

51. Chandler, J., Hohenstein, P., Swing, D.A., Tessarollo, L., and Sharan, S.K. 2001. Human BRCA1 gene rescues the embryonic lethality of Brca 1 mutant mice. Genesis. 29:72-77.

52. Yang, Y., Swaminathan, S., Martin, B.K., and Sharan, S.K. 2003. Aberrant splicing induced by missense mutations in BRCA1: clues from a humanized mouse model. Hum. Mol. Genet. 12:2121-2131.

53. Sharan, S.K., et al. 2004. BRCA2 deficiency in mice leads to meiotic impairment and infertility. Development. 131:131-142.

54. Healy, E., et al. 2001. Functional variation of MC1R alleles from red-haired individuals. Hum. Mol. Genet. 10:2397-2402.

55. Marth, J.D. 1996. Recent advances in gene mutagenesis by site-directed recombination [review]. J. Clin. Invest. 97:1999-2002.

56. Fries, R.S., et al. 2004. Neuroendocrine transcriptome in genetic hypertension. Multiple changes in diverse adrenal physiological systems. Hypertension. 43:1301-1311.

57. Sinha Hikim, A.P., and Swerdloff, R.S. 1993. Temporal and stage-specific changes in spermatogenesis of rats following gonadotropin deprivation by a potent gonadotropin-releasing hormone $(\mathrm{GnRH})$ antagonist treatment. Endocrinology. 133:2161-2170.

58. Hikim, A.P., Amador, A.G., Klemcke, H.G., Bartke, A., and Russell, L.D. 1989. Correlative morphology and endocrinology of Sertoli cells in hamster testes in active and inactive states of spermatogenesis. Endocrinology. 125:1829-1843.

59. Cruz-Orive, L.M., and Weibel, E.R. 1990. Recent stereological methods for cell biology: a brief survey. Am. J. Physiol. 258:L148-L156.

60. Kennedy, B., and Ziegler, M.G. 1990. A more sensitive and specific radioenzymatic assay for catecholamines. Life Sci. 47:2143-2153.

61. Tanaka, N., et al. 1996. Transthoracic echocardiography in models of cardiac disease in the mouse. Circulation. 94:1109-1117.

62. Collins, K.A., Korcarz, C.E., and Lang, R.M. 2003. Use of echocardiography for the phenotypic assessment of genetically altered mice. Physiol. Genomics. 13:227-239. 